\title{
对人文科学所遭受抨击的回应
}

\section{Iain HAY}

(澳大利亚弗林德斯大学人文、艺术与社会科学学院, 阿德莱德 5001)

\begin{abstract}
摘 要: 长期以来, 不少人站在功利角度,对人文科学进行了颇多抨击。本文意欲对此做出有理有据的反击。首先, 文章概述了人文科学在政界、民间和实践领域所遭受的非议,并寻找出主要的批评者及其拥护者。其次,作为申辩 内容的一部分, 本文详细阐述了人文学科的核心优势。这些优势涵盖了哲学和实用科学领域, 包括为知识创造提 供批判性视角, 使生活丰富多彩, 培养应对未来不确定性和复杂性的技能, 以及增强理性和维持大学机构的收人和 信誉度。最后, 本文提出人文学者不仅要开展相关学科里非常重要的理论和实证研究, 还必须将捍卫和保护人文 科学纳人到专业活动中来。
\end{abstract}

关 键 词: 艺术; 批评; 捍卫; 人文; 文科; 实用

《狄德勒斯》专门用了一期来讨论人文学者到 底是做什么的, 美国文学评论家奚立斯・米勒在其 中评述到“如果人文科学的社会价值没有成为一个 饱受争议的话题, 那么也就用不着做这样一期《狄 德勒斯》了”(Miller, 2014: 19)。正是认识到这种质 疑的普遍性, 本文决定对此做出一些回应。文章首 先概述了人文及相关学科(如人类学、经济学、人口 学、地理学、历史学、语言学、文学、社会学和哲学) 在哪些方面饱受非议, 并指出背后具体的批评者及 其同谋者(包括人文学者自己)。对人文科学在个体 和社会价值方面的质疑, 正是受到了孤婳寡闻的功 利主义思想的影响, 所以文章紧接着讨论了人文科 学到底能为社会提供什么价值。这些事实证据是 为人文科学辩护的核心内容。最后, 文章就如何应 对一些人对人文科学的非难, 提出了若干切实可行 的建议。本文认为适当的还击也是现代大学里人 文学科的一项重要职责。总之, 本文本质上是对人 文科学所遭受的功利性批评做出的务实回应。

\section{1 备受抨击的人文科学}

正如世界各地的很多作者所指出的那样, 人文
科学正遭受到来自多个领域的压力一一即便在一 些世界最知名的大学里亦是如此(Phamotse et al, 2008; Lewin, 2013; Mittelstrass, 2015; Preston, 2015; Sawa, 2015)。尽管这样的抨击早已有之,但 其激烈程度及波及范围正在不断扩大, 有从现实层 面(如人学学生的专业选择)上升至意识形态的趋势 (如政界人物对大学的洗劫)。

有证据表明, 长期来看, 人文学科的学位在灵 活性和职业发展发面具有一定的优势, 特别是在当 前就业前景越来越不可知的情况下。尽管如此, 许 多学生依然选择或被迫功利性地选择攻读可立即 适应职业市场的就业导向性学位(Institute for the Future, 2011; Else, 2014; OECD, 2016; OECD Labour and Employment Ministerial Meeting, 2016)。 学生的学业选择受到家长、学习顾问以及“善意”友 人的影响,这些人在经济大环境不好的情况下都急 于提出具有经济理性的忠告。对于一些家庭条件 并不好的学生来说, 学习人文学科就像海市傆楼一 样虚无缥组。正如比洛特所言, “大学教育成本上 涨, 文科教育对工薪阶层和中下等收人家庭来说已 无法承受。这些家庭的孩子被迫放弃自身需求转 而寻求职业导向的教育”(Billotte, 2012)。高涨的高

收稿日期: 2017-10-24; 修订日期: 2018-03-25。

作者简介: Iain HAY,澳大利亚弗林德斯大学教授,任 IGU 副主席, 主要研究方向为政治地理学, 超级富豪与社会不公, 研 究伦理等,E-mail: iain.hay@flinders.edu.au。

(1) 译者注: 《狄德勒斯(Daedalus)》是 1955创办的一本学术期刊, 其前身是《美国艺术与科学学院学报》。

引用格式: Hay I. 2018. 对人文科学所遭受抨击的回应[J]. 地理科学进展, 37(3): 317-326. [Hay I. 2018. Defending letters: A pragmatic response to assaults on the humanities[J]. Progress in Geography, 37(3): 317-326.]. DOI: 10.18306/dlkxjz.2018.03.002 
等教育成本意味着多数学生必须在选择教育时慎 之又慎。例如《卫报》曾报道, 在英国政府决定将 2013-2014 年度的学费上限提高到原来的 3 倍, 即 6000 英镑(约为 11500 澳元)后, 英国成为了世界上 本科教育平均学费最高的国家, 超过美国的 5300 英 镑(约 10000 澳元)和日本的 3300 英镑 (约 6200 澳 元)(Walker, 2015)。近期澳大利亚国会预算办公室 发布的一份独立报告显示(PBO, 2016: 23), 预计到 2026年, 澳大利亚联邦的全日制学生在未实施学费 管控的地区,完成 3 年学位课程所需花费的平均学 费将超过 50000 澳元。面对这样的巨额成本, 学生 倾向于选择易于就业的学科也就不足为奇了。

除了学生抱有职业和经济方面的实用主义思 想外, 全球保守派政客的态度也令人担忧。如, 2015 年因丑闻下台的澳大利亚城市与建成环境部 部长杰米-布里格斯(Jamie Briggs)曾有如下表现:

……对他认为 “荒谬”和浪费政府拨款的人文科学进行 过抨击……这位政坛新星声称: “我们要在澳大利亚开展科 学研究, 但我们希望把研究重点放在真正有意义的事情 上”。那些被认为 “荒谬” 的研究主题包括性别、伊斯兰教和 一些哲学方面的课题(Bonnell, 2014)。

再来看看其他地区。2015 年年末, 日本教育部 部长 Habukan Shimomura 曾给国内 86 所国立大学 写信, 呼吁他们采取“积极行动以废止(社会科学与 人文学科的)研究机构或将这些机构转向服务其他 更符合社会需求的领域”(Grove, 2015)。在此情形 下,很多高校还纷纷表示将遵照执行(Dean, 2015)。 在英国,保守派教育大臣尼基・摩根(Nicky Morgan) 声明, 可以使年轻人有充足选择机会、能够打开所 有职业的大门的学科是科学、技术、工程和数学(即 STEM 类学科)(引自 Dean, 2015)。在美国, 共和党 总统候选人、参议员马尔科・卢比奥(Marco Rubio) 在 2015 年语带讯讽地提到, 学生们应当提前知道 “是否值得花费 40000 美元主修希腊哲学, 因为希 腊哲学家的就业市场很窄” (引自 Scalia, 2015)。尽 管这些举动令人堪忧, 但和近期弗罗里达州和德克 萨斯州通过立法来鼓励学生优先选择主修科学、技 术、工程和数学(STEM) 学科而非人文学科的做法 相比,简直是小巫见大巫！(Scalia, 2015)。

不论是不是完全由于务实功利主义和意识形 态功利主义的影响, 但在教育方面极其有限的、且 不断削减的公共与私人投人的确进一步恶化了人 文学科的发展。在美国, 国家对人文学科的拨款从 1979 年的 4 亿美元(以 2016年美元计)下降至 2015
年的 1.5 亿美元(以 2016年美元计)(American Academy of Arts and Sciences, 2015)。美国艺术与科学学 院在其发布的报告《问题的核心》中揭示, 来自联邦 政府的拨款从 2005 年的 30\%降至 2011 年的 $20 \%$ 左 右 (American Academy of Arts and Sciences, 2013: 40), 而同期联邦政府对数学、物理科学、生物科学、 医药科学和工程学的资助则保持在 $60 \%$ 70\%的水 平, 然而也没有公开证据表明这些投资得到了回 报。特拉斯等人的研究表明,在 2010 年, 美国联邦 政府只有 $0.45 \%$ 的研究经费是流向人文学科的; 而 在 2007-2013 年间, 欧洲委员会基金拨给社会科学 和人文科学的经费只占总额为 555.1 亿英镑科研经 费的 1.06\%(Terras et al, 2013)。

人文科学不仅在国家层面获得资助甚少, 在一 些学校机构中,STEM类学科的发展也抢占了大量 科研经费, 使人文学科经费更加匮乏。一些 STEM 领域的工作确实比人文学科花费大,正如美国关于 “高等教育成本、产出和义务”的三角洲项目执行总 监简・威尔曼(Jane Wellman)所观察到的:

大学之所以还能维持, 是因为 “交叉补贴”, 即成本低廉 的课程补贴和资助成本高的课程。成本较低的课程包括英 语和社会学, 成本昂贵的课程包括医学。这意味着在实际 的高等教育资助方面, 英语和社会学通过招生获得收人, 但 收人并没有用于这些学科自身的科研而大部分是让他们自 筹资金; 就我自己评审的项目来看,一些机构普遍将从教学 中获取的“利润”转向用于资助一些更为昂贵的研究领域(引 自 Newfield, 2010)。

因此,换句话说,与正统新自由主义价值观所 提倡的注重效率恰恰相反, 成本又低产出又高的人 文科学得到的回报却是越来越少的资助。

除此以外, 还有日常生活中没完没了的对人文 科学的侮辱, 比如则所墙壁上的涂鸦(则纸器上方 写着“文学学士学位在此, 请拿走”); 还有一些不了 解事实、瞎贫嘴的广播节目对人文科学的贬损; 以 及一些博文和保守派杂志(如 Hopkins, 2014)也会 流露出对人文学科的不恭。这些仅仅只是在公共 场所出现的种种非议人文学科的一小部分。这些 不当言论的影响力却是非常巨大的, 以至于一些人 文学科的支持者沉默了, 或者改变了自己公开言论 中的表述。我们可以从关于美国里海大学文理学 院院长唐纳德・霍尔(Donald E. Hall)的一则轶事中 略窥一斑:

我同坐在我身旁的一位男士开始交谈起来,他谈起他 和我一样, 是阿拉巴马大学的文学士毕业, 只不过比我早 3 
年。我问他现在从事什么行业,他表示他拥有一家很成功 的大型保险企业。我又问他在阿拉巴马学的什么专业, 他 说: “是丝毫没用的东西, 和我现在的成功没有半点关系, 就 是政治科学。”我刚好也在同系获得的政治科学文学学士学 位, 我说: 你怎么能认为每节课都要学习如何分析数据、编 撰长达 20 页的研究报告, 提出并论证自己的观点、在课堂讨 论时与同学达成共识等等,这些和你现在职业上的成就没 有半点关系呢? 我认为这些和我之所以能成为一名成功的 行政管理人员息息相关。听完他脸红了, 回答说知道我的 话确实有理, 但他已经习惯了为自己所学的东西道歉, 以至 于最终彻底放弃尝试为其争辩, 并和别人一样, 说学文科毫 无用处。他甚至承认, 他每年都向阿拉巴马大学文理学院 捐赠 5000 美元, 以表达对学院的感激之情(他并未向足球队 捐赠过一分钱),但他却从未向朋友们提过此事(Hall, 2013)。

人文科学在多个领域备受非议, 从学生的专业 选择到政治家的公开演说,再到我们自己的日常对 话, 比比皆是。面对如此猛烈的批评, 人文学科究 竟能做些什么? 对那些怀疑人文科学价值的人, 我 们又可以说些什么呢?

\section{2 人文科学可以贡献什么? 知识、文 化、经济和社会的批判性视角}

人文科学的从业者与其他文科行业的人员一 样, 他们的核心工作是分享和创造知识, 加人(学术) 团体、不解审视并挑战现有认知、不断考证实证发 现、重构现有理论。这种知识的创造可以是为实际 所需, 也可以纯粹是为知识本身。但后者在当代大 学和政治界所获得的支持已越来越少了(Matthews, 2013)。对知识本身和知识生产的吒问能催生出有 关权力关系的深刻的批判性思考, 而知识本身其实 也代表了某种权力关系(Foucault, 1988)。权力和知 识之间究竟是什么关系? 权力来自哪里? 谁有凌 驾于知识之上的权力? 知识到底是在为谁服务? 这些都是非常重要的问题, 不说要加大关注力度, 但至少应当持续关注。

当今人类社会的发展已达到人类历史上无与 伦比的生活水准和知识水平, 但同时也面临前所未 有的发展不公和经济环境危机。对于那些引领我 们人类社会发展至今, 或功不可没、或罪大恶极的 理念或实践, 人文科学也可以提出一些有益的见解 (Starr, 2013; Hay \& Beaverstock, 2016; or humanities work addressing global inequalities at the University of Manchester)。人文科学研究者还尤其擅长审
视我们日常生活所在的这个已习以为常的世界。 例如, 通过科学的计量分析, 可以发现人们对许多 事物的惯常认知与由分析得出的真知之间存在着 巨大的差异。如麦克劳德对猎鸭人破坏环境这一 常见看法就提出了质疑,认为这些猎鸭人为保护和 培育湿地、守护鸭群和其他野生动物发挥了重要作 用(McLeod, 2004)。布林德利的研究强调澳大利亚 郊区住房的那些后院,在当下这个亟需城市政治团 结的政治经济环境中,具有重要的社会意义(Brindley, 1999); 而海德和缪尔则评估了后院更具广泛意 义的环境重要性(Head \& Muir, 2013)。海氏和马勒 对慈善到底能带来多少 “善行”表示怀疑, 指出了大 规模 “捐赠”所产生的一系列社会、经济和地理问题 (Hay \& Muller, 2014)。

\section{1 帮助加深对他人和世界的理解}

我们或许无法完全体会别人的生活,但人文科 学可以使我们更好地分享经验, 了解他人的世界。 一个极好的例子是,一些土著语言对我们认识人与 环境关系中的文化特殊性极具启发。例如语言学 家迈克尔・克里斯蒂在他有关澳大利亚雍古族 (Yolnu)的研究中吐露:

我试着在脑海中将雍古族语(Yolngu Matha)中的物体名 称排成某种体系, 但没有成功。我本以为像“植物”和“动 物”这类词语之间的区分是“天然”的, 是本就存在的, 不是 人类出于认识世界而采取的某种人为行为。但在雍古族语 中, 没有与“植物”或“动物”对应的词(Christie, 1992)。

克里斯蒂进一步发现, 雍古族语中的一些分类 名称很少能让英语母语的人觉得毫无争议、无需解 释的。尽管一些人对此感到不安,而一些人觉得兴 奋, 但冊庸置疑的是, 人文科学的这类研究, 对思考 客观现实与认知世界以及时空之间更广阔的联系 等相关问题,的确提出了很多非常深刻且需要不断 研究的议题(Dear, 1988; Soja, 1989; Sayer, 2000; Smith, 2006)。

另一个大相径庭的例子是, 有关战争的描述 (和摄影)可以让未参与过战争的人了解到战争冲突 可怕的一面。凯特-麦克洛克林的近作《战争创作： 从伊利亚特到伊拉克战争的文学表现》, 阐释了近 3000 年来,作家们以各种方式所揭示的不同形式的 冲突所带来的暴力、混乱和痛苦 (McLoughlin, 2014)。英国反战诗人维尔浮莱德・欧文(Wilfred Owen)从身为一个战士的个人经历出发, 令人信服 地描述了第一次世界大战对身体、道德和心理所造 
成的创伤。其著名诗作 S.I.W.(1920) 以不到 350 字 的篇幅有力描述了战士蒂姆所面对的恐怖的战争, 读者能够从诗作的病态结尾中体会到这种沉重:

他们将他和他牙齿亲吻过的枪口埋葬在一起,

母亲真心地写道: “蒂姆含笑离开”。

现代社会中的战争更加背离人性, 人文科学对 其所进行的审视、解读和传播(如通过教学)至少为 社会提供了一点有人性的反战呼声和政治情绪。 通过人文科学的文字工作, 可以传承对人与人之 间、文化与文化之间的理解, 从而使我们人类产生 更和谐的同情心, 更好地学习如何认识别人所认知 的世界, 谦逊地意识到别人可能观察到了我们所没 有看到的世界, 甚至可能比我们自己所认知的“更 准确”(Fulbright \& Tillman, 1989: 217)。

\section{2 使生活更加丰富和有意义}

人文科学通过培养我们在创造性和建设性方 面的潜能, 丰富了我们的生活。它让生活更加美好 丰富、健康快乐、充满价值和乐趣。人文科学可以 教授我们很多技巧和能力, 使我们的人文精神和特 性可以从繁重无聊的日常工作中释放出来, 得以升 华。因此, 人文科学是人之为人的重要组成部分。 威廉·德莱塞维茨在他的近作《优秀的绵羊: 美国精 英教育的劣势和通往有意义生活的道路》中提出了 文科教育的终极目标是:

……帮助你学习如何在工作和事业之外进行有深度和 广度的反省: 为了成为一个合格的公民, 为了与他人和谐相 处, 以及最终为了使自己变得更加坚强、自由和富有创造力 (Deresiewicz, 2014: 155)。

除了培养公民意识和自我意志外, 人文科学还 可以通过培养相关能力来丰富我们的生活, 使我们 具有发现身边事物的价值和判定事物重要性的能 力。正如美国最高法院法官戴维・苏特(David Souter)在 2010 年哈佛大学毕业典礼致辞中所言: “法官 必须在宪法所允许和珍重的、然而又往往存在相互 竞争的价值观中做出选择, 比如在自由和平等中做 出选择, 并且, 法官所做的选择不应是基于权衡的 结果, 而应是基于事物本身的意义和价值所在” (Souter, 2010)。苏特的评论进一步支持了同年哈佛 大学校长德鲁・吉尔平・福斯特(Drew Gilpin Faust) 就创造价值所提出的观点:

我们都受到了信息的轰炸。信息爆炸是界定当下全球
新知识经济的一个要素……人类面对这样海量的信息应如 何处理? 如何消化和评估? 如果我们要发展知识经济, 那 么首先应该要理解什么是干扰的噪音,什么才是真正的知 识,或者也可以说,什么是信号一以区别于单纯的信息。 仅仅把教育当成经济增长的工具导致我们忽视了这方面能 力培养的重要性。它忽略了我们都只是(信息的) ${ }^{2}$ 解读者这 一事实; 忽略了有些事情并不关乎 “事实”, 而是关乎理解和 意义(Faust, 2010)。

\section{3 增强适应未来不确定性的能力}

人文科学可以培养我们适应不可预测(和“全 球化”)的未来。第二次世界大战后不久,埃莉诺・ 罗斯福(Eleanor Roosevelt)在美国史密斯学院演讲 时问道: “我们生活在一个不断变小的世界里,我们 必须与有着不同文化、不同习俗爱好和不同宗教信 仰、遵循不同法律制度、说不同语言的人近距离相 处, 我们要为这样的生活做哪些准备呢?”(1949, 引 自 Christ, 2005)。受国际人口迁徙、全球贸易、电子 通讯普及和全球流动性等因素的推动,不同人类文 明之间的接触越来越频繁, 60 年前罗斯福夫人所提 出的问题在今天显得愈加具有现实意义。最近美 国艺术与科学学院也强调了这一点, 指出: “我们参 与全球经济,要了解不同的文化,要知道不同文明 可能持有不同的观点。人文和社会科学可以教授 我们如何理解、解读和尊重不同文化的共同点和差 异性” (American Academy of Arts and Sciences, 2013: 57)。当今社会比人类历史上任何一个时期 都更加需要这种能在不同文化、地理、法律、社会中 自由切换的毕业生和社会公民。社会对这些机敏 的人文学科毕业生持续不减的广泛需求, 绝不仅仅 是因为 “地球村”里越来越密切的交往, 也可能是交 往中所带来的难以预料的后果。比如, 2001 年的 911 事件后,阿拉伯语和伊斯兰教历史方面的专家 突然发现他们变得紧俏起来, 因为各国都需要咨询 他们有关与穆斯林国家公关外交方面的事宜 (Douglas \& Neal, 2013)。

在社会、文化、技术变革和人类活动的其他领 域,也都离不开人文科学。例如,改善国际贸易关 系需要精通新贸易伙伴的语言、文化和地理知识; 数字化的发展要求用新的方法在商业中建立互信; 要有效应对老龄化问题和流动性越来越强的人口 就意味着要了解新兴的家庭结构、基于不同个体的 生活经验和生活能力、住房设计和合理的经济扶持

(2) 括号里的内容为译者为精确转译而添加的部分。 
机制等; 国际维和行动及其在当地所实施的内生发 展策略要想卓有成效, 也必须了解要找谁参与制定 政策, 怎么找和在哪儿找。哪怕是应对人类所引起 的气候变化这一问题, 也绝不仅仅是单靠自然科学 所能解决的, 而同样需要人文科学。例如需要人文 科学的工作来确保公众对这一问题及其修正措施 有广泛的认知和理解。总之, 培养适应和应变能力 需要人文科学。

\section{4 在 STEM 学科中增强理性同时保持人文精神}

人文科学以各种方式扶持和培育着 STEM 学 科的发展。

(1) 人文科学让人们得以了解科学意义存在的 背景及其权力 (义务)。正如评论家在评论最近英国 的社科运动报告《事关人人》(Campaign for Social Science, 2015)中所提到的那样: “必须重复一个基 本的但又常常被遗忘的真理, 那就是科学是在特定 的社会经济背景下运作的。如果我们不了解人类 行为、态度和组织的动向, 科学也走不了太远” (Wilsdon \& Walker, 2015)。例如, 当今人类延年益 寿的科技手段发展已远远超乎了之前的想象, 但这 也带来了许多问题, 甚至使我们不得不重新全盘考 虑对待安乐死的态度和法律。全新的生殖技术在 家庭、父母、性爱、婚姻等领域也引起了重大的道德 和现实问题。与传统的物种选择或杂交等生物技 术方法不同, 使用现代生物科技的转基因食品也给 野生生物、广大的自然环境、人类健康、消费者信誉 以及未来的社会经济等带来了潜在的风险; 转基因 技术必须权衡它在提高农作物产量、环境效益(如 减少农药使用量)和人类健康(如提高营养水平)等 方面的利弊(McLean, 2005)。在神经工程学领域, 人与人之间以及人与动物之间的脑对脑接口 (Brain-to-Brain Interface, BBI) 已经得以实现, 可以 通过一个大脑对另一个大脑进行无语言直接沟通 并发出物理指令。正如一些研究所认为的那样, 这 种技术的伦理关系是错综复杂的 (Trimper et al, 2014)。譬如, 到底谁将为如此行为负责? 是接收 命令的执行者还是发布命令的谋划者? 如果在使 用脑对脑接口的过程中产生了新的想法, 那么这个 想法在合法性上应该归属于谁呢? 另外, 由于一个 人的大脑与另一个人或众多人的大脑相互对接, 那 么这个人该如何定义自己的身份呢? 因此, 有人文 科学学者曾提到(Hohendahl, 2005):

科学、医学和工程是不可或缺的实用学科, 它们可以改 变人类世界及其与自然的关系。而人文科学则是决定哪些
事是可以做的学科, 它可以评价出哪些才是我们应该追求 的目标, 哪些项目是合乎人性的……

(2) 有些比较权威的人士认为人文科学有助于 培养更好的科学家、工程师和医学从业者(反之亦 然)。例如, 诺贝尔化学奖得主美国生物化学家托 马斯・R ・切赫(Thomas R. Cech)认为 : “即便是对那 些将来永远也不会使用微积分的人而言, 数学也被 认为是一项很好的大脑运动; 同样, 阅读、学习历 史、语言、音乐等其他非自然科学的内容, 也会磨练 一个科学家感知和解读自然世界的能力 (1999: $210)$ ”。人文科学还可以帮助医学治疗。例如, 2015 年的《医学人文学(Medical Humanities)》期刊 就通过一组特别的诗歌、散文和研究型文章来揭示 “医疗服务的过程、公共医疗政策的形成、以及生病 的经历和护理病人的过程......”等等。该刊物通过 多视角体察病人和医务工作者双方的经历和情感 过程, 以帮助培养出更多具有人道主义精神的医护 人员,使他们能跳出自身经历局限而基于医患双方 的生活体验和理解去处理问题。另外,近期该期刊 的一篇文章也发现, 医学人文学研究非常有助于协 调失衡的医患关系(Garden, 2015)。

(3) 除了提高科学家的批判能力以外, 自然科 学与人文科学的结合还可以产生巨大的生产力。 哲学家玛莎·努斯鲍姆发现, “中国、新加坡等之前 忽视人文科学发展的国家, 正在积极地推广人文科 学, 因为这些国家意识到想象力对于促进创新和提 高创造力至关重要, 而培养想象力则需要学习文 学、电影和其他艺术......” (Nussbaum, 2010)。自然 与人文科学完美结合的一个现实案例, 就是美国的 苹果公司。前苹果首席执行官史蒂夫 ·乔布斯在 2011 年推出 $\mathrm{iPad} 2$ 时, 就曾说过一句被广为报道的 话: “在苹果的 DNA 中, 光有技术是不够的, 它是与 自由艺术的结合、是与人文精神的结合, 这样才创 造出让我们心为所动的产品”(引自 Lehrer, 2011)。 乔布斯无疑是将人文科学与技术相融合的大师; 而 苹果的这一模式也显然表明,通过集合科技和人文 在不同领域的优势, 自然科学与人文科学是完全可 以产生出极具生产力的协同效应的。

(4) 除了苹果公司的事例之外, 还有很多证据 表明人文学科的毕业生同样具备其他有关科技、就 业和商业方面的技能。例如, 2012 年对 652 名美国 出生的首席执行官和产品工程负责人的调查显示, 近 60\%的人拥有文科学位(Wadhwaet al, 2008); 另一 项对伦敦证券交易所前 100 上市公司(100 FTSE 
companies $)^{3}$ 首席执行官的研究显示, 其中 $34 \%$ 的 $\mathrm{CEO}$ 们是艺术、社会科学或人文科学相关专业毕 业, 而理工科(STEM)毕业的人仅为 $31 \%$ (Batstone, 2012; Terras et al, 2013)。对于了解人文科学的人来 说, 这些结果一点也不意外, 因为沟通、解读、批判 性和建设性思维、解决复杂问题、重视伦理和团队 合作正是全世界的人文科学所要培养的专业素 养。而且以目前的形势来看, 未来的职业只会越来 越需要人文科学专业所强调的这些能力和其他素 养。因为未来那些我们还想象不到的新兴职业会 对思维的广度、灵活性和批判性思考能力要求越来 越高,而不太可能是线性的、机械的工作。

(5) 大体来说, 现在地球上已经很少有什么地 区或自然系统是完全 “自然” 的了, 自然界几乎都受 到了人类的影响(沙漠、珊瑚礁、大气等), 理解和治 理这些问题既需要科学、技术、工程、数学等方面的 技能, 也需要人文科学方面的专业知识。

\section{5 支撑大学等机构的收入和信誉}

人文学科还可以帮助支撑大学的收人和信誉 度。人文学科作为大学的科目已有 500 1000 年的 历史, 并且一直是大学的核心。正是因为其在人文 学科领域的权威性, 大学才得以称之为大学(universities)而非技术学院 (technical institutes or colleges)。正如美国批判教育学的代表人物亨利・吉鲁所 委婉阐述的那样, 从大学与人文学科的这种渊源上 我们可以得到的一个教益是: “人文学科的规模越 缩小、越私有化、越商品化, 高等教育就越会背离其 初衷; 因为大学一面说着要为年轻人的未来投资, 一面又不给年轻人提供什么理智、公民和道德方面 的培养” (Giroux, 2011)。也就是说, 如果真像很多 大学的校训所描述的那样, 要让学生得到全面发 展, 那么大学就必须提供渠道以赋予学生在理智、 公民和道德方面的能力。

人文学科可以吸引到一批对价值、伦理、公正、 道德和平等比计量、机械、市场和金钱更感兴趣的 学生, 这其实也可以维持大学的收人和信誉度。换 句话说, 这些文科生可以为那些还在为发工资而发 愁的大学提供宝贵的人学率(Lewin, 2013), 维持一 定的、多样化的收人来源, 这说起来真是具有讽刺 意味。

\section{3 人文学者需要采取行动提升人文科 学地位}

了解和赞颂人文科学当然十分重要, 但鉴于本 文之前所提到的那些基于功利主义思想和带有意 识形态色彩的抨击, 为人文学科辩护需要采取更加 强硬的行动。捍卫人文科学也是当代人文学者义 不容辞的一项责任和义务。只有努力宣传人文科 学,才可能提振人文科学的发展。但正如美国艺术 与科学学院所认为的那样, 不加付出努力, 人文科 学的复兴是不可能到来的 (American Academy of Arts and Sciences, 2013: 40)。那么这要求我们做出 什么努力? 我们可以采取哪些切实可行的策略?

(1) 需要修改或增设大学的课程和学位, 通过 系统地整合大学的辅修、核心和通识课程, 来更好 地融合人文学科和理工学科。德国著名剧作家贝 托尔特. 布莱希特早在半个世纪前就曾提出有力的 辩护(Bertolt, 1964: 73):

艺术与科学的工作方式不一样一一这个我同意。但是 ……我认为, 这个世界上最伟大和最复杂的事情, 如果不借 助一切可能的认识工具就不能够被人类充分认识。

令人振奋的是,一些地区已经在谨慎地开展这 方面的工作。例如, 2015 年 12 月,美国国家科学 院、美国国家工程学院和美国国家医学院的高等教 育和劳动力委员会召开了题为 “融合人文艺术与理 工科教育”的研讨会。会议的一个目标就是改革现 有课程, 设置更具融合性的课程体系, 从而更好地 培养学生成为一名公民和工作者,让他们为“负责 任地解决社会所面临的巨大挑战” 做好准备(National Academies of Sciences, Engineering, and Medicine, 2015)。此外, 加利福尼亚大学伯克利分校在 其文理学院也开设了一套被冠名为 “伟大的思想” 的课程(UC Berkeley, 2016)。这套课程旨在探讨一 些“概念上错综复杂、超越某一具体学科领域的议 题, 其中设有一些独特的课程, 比如神经系统与佛 学; 以及“感知、感性和科学’, 以探讨哲学与社会科 学方面的问题”(Romano, 2016)。

(2) 人文学者和人文领域的其他从业者要努力 多做工作以提高公众认知、影响公共政策。关注人

(3) 译者注:伦敦金融时报 100 指数(或伦敦金融时报 100 种股价指数), 简称富时 100 指数(FTSE)。创立于 1984 年 1 月 3 日,是在伦敦证券交 易所上市的最大的一百家公司的股票指数。它由伦敦金融时报编制。该指数是英国经济的晴雨表,也是欧洲最重要的股票指数之一。相关 的股票指数包括富时 250 指数(除了 100 家最大的公司以外接下去的 250 家最大的公司的股票指数) 和富时 350 指数(富时 100 和富时 250 指 数的结合)。 
类所面临的一些“重大挑战”也许最容易产生效果， 如围绕纯净的空气、水、粮食、健康、能源和普世教 育等方面的供给问题(American Academy of Arts and Sciences, 2015), 但还有数不胜数、千变万化的 其他领域值得研究。比如, 生活标准该如何合理分 配(Gruen 等, 2011)? 与巨大的等足类动物、叮蛰动 物等 “笨拙” 的非人类生物相处有哪些伦理问题 (Ginn 等, 2014)? 在一个饱受恐怖主义袭击和备受 监视的时代, 个人隐私的权力在哪里 (de Rosa, 2003)? 出口活体动物有什么道德伦理问题(Coghlan, 2014)? 怎样才算死得有尊严(Gentzler, 2003)?

(3) 非常有必要收集和积累人文领域中一些成 功的个人或集体的、定性和定量数据, 以及能反映 人文科学重要性的实例, 并将这些信息用于地方、 国家和国际等各个层面有关支持人文科学的公关 活动中去。这样的活动有很多, 有个人身体力行 的、也有通过创立专门的机构来为人文科学摇旗呐 喊的。比如, 2015 年新西兰梅西大学文科学位督导 理查德・肖(Richard Shaw)在校内作了有关揭开文科 学位神秘面纱的讲座; 还有英国社会科学院发起的 影响深远的社会科学运动; 以及由澳大利亚、加拿 大、英国和美国的人文学者和从业者所组成的国际 4Humanities 运动, 它通过 “创造性地使用数字科技 来推动人文科学的研究与教学” (4Humanities, 2015)。

(4) 人文学者及其所在机构有必要与记者加强 联络, 而不是避开那些难以控制的媒体, 并且要通 过媒体、公开讲座和其他方式多在公众场合抛头露 面, 促使公众积极关注人文科学的发现、洞察和解 读。正如美国艺术与科学学院在其具有里程碑意 义的报告—《问题的核心》中所提到的那样, “公 众对人文科学的评价, 需要靠一步步打破学术知识 的自我封闭、加强与外界的联系来加以巩固”

(American Academy of Arts and Sciences, 2013: 43)。

(5) 我们必须努力重拾自信一一这里 “我们”指 的是人文学者、理工领域(STEM)的从业者以及家 长和大学的行政人员一一要确保在我们的日常交 谈中、在与学生的交流和与同事的对话中传递 “正 面的信息”。正如前文提到的美国里海大学文理学 院院长唐纳德・霍尔所说的, 以及像麦肯锡公司评 论员在 2011 年发表的评论那样, “现在我们所有人 都是营销者”。因此, 要确保为学校的教职人员、学 生和校友提供必要的手段和数据以做好这项营销 工作, 并保证要他们所传递的信息是与其他人的言
行相吻合的。

(6) 我们必须重新审视大学的规章制度, 以更 合理地评价人文科学的工作。譬如,我自己所在的 弗林德斯大学跟其他许多学校一样, 在过去几年逐 渐采取了“偏向自然科学”的绩效考评模式,如将多 人合作论文(这在理工科(STEM)中更为普遍)的工 作量视为与单一作者论文的工作量等同, 尽管这显 然与澳大利亚联邦对学校的评估要求不符。而我 自己所在的环境学院 — 一个横跨自然科学与人文 科学的学院一若不是遭到坚决抵制, 竟差点实施 了与上述论文考核截然相反的著作绩效考核标准, 即合著著作的工作量, 却必须按照作者数量来分 摊。此外,科研经费成为衡量科研人员工作量和职 称晋升的重要指标,却完全没有注意到人文学科与 理工学科在资金需求上的显著差异。尽管举的都 是我自己所在科研机构的例子, 而且大学部门的奖 励体系差异性也很大, 但对任何这类不利于人文科 学发展的改变, 我们都需要密切留意并奋力抵制。

\section{4 结论: 人文科学可以贡献很多,也 还有很多工作要做}

由于人文科学备受攻击, 人文学者们还要做更 多的抗争。政治家公开贬低我们的工作; 大学将我 们边缘化, 却又利用人文学科来吸引招生和申请科 研经费; 家长和职业顾问鼓励准大学生避开人文学 科,而选择更切合实际、更有利可图的领域; 媒体流 传着让人忧心的盲目比较人文与自然科学价值的 文章。或许是厌倦了外界对人文科学没完没了的 抨击, 一些人文学者开始向这些言过其实的报道妥 协, 并退缩至个人的学术领域, 不敢抵制與论的浪 潮, 屈服于每天都要面对的斗争压力, 或因准备不 足而难以坚持这场长期的战斗。然而, 尽管存在着 大量负面的传闻和与事实不符的报道,但事实上人 文学者可以为现代大学及其赞助者做出很多杰出 的贡献, 无论在哲学和还是实用科学领域。例如, 为知识生产提供批判性视角, 使生活丰富多彩, 帮 助年轻人获得必须的能力以应对充满不确定和日 益复杂关联的未来, 传承人文精神, 增强理性, 支撑 大学机构的收人和信誉。显然, 人文科学实际所具 有的无形和实用价值, 与社会对其的认知之间, 存 在着明显的脱节。我们人文科学研究的意义对他 人来说并非是不言自明的。这样的认知差距不可 能靠其他人来弥合, 而显然要靠人文学者们自己。 
因此, 不论人文学者自己内心多么清高、多么不屑 于回应, 我们都不得不学习如何更好地应对这些持 续地、甚至可能变本加厉的、基于实用主义者自己 的价值观和重要性判断所做出的对人文科学的非 议。所以, 人文学者不仅要继续进行相关学科里非 常重要的理论和实证研究, 还要在自己的专业工作 中增加一项内容, 即捍卫人文科学的尊严。这样的 努力不仅是为传承我们的工作, 还可以通过捍卫人 文科学的过程加深反省, 更好地了解我们自己、我 们所做的工作以及这样做的原因。总之, 人文学者 们还有很多工作要做。

致谢: 本文原文(英文)2016年7月7号发表于 Journal of Higher Education Policy and Management, 感 谢原出版商 Routledge 公司授权在《地理科学进展》 发表中文版本; 感谢河海大学公共管理学院李禕副 教授将英文译成中文。

\section{参考文献(References)}

4Humanities. 2015. Mission[EB/OL]. 2015-04[2018-03-25]. http://4humanities.org/mission/.

American Academy of Arts and Sciences. 2013. The heart of the matter[R]. Cambridge, MA: American Academy of Arts and Sciences.

American Academy of Arts and Sciences. 2015. National Endowment for the Humanities (NEH) Funding Levels[R/ OL]. 2015-10[2018-03-25]. http://www.humanitiesindicators.org/content/indicatordoc.aspx?i=75\#fig379.

Batstone M. 2012. In defence of the humanities[N/OL]. The Guardian. 2012-01-11[2018-03-25]. http://www.theguardian.com/higher- education- network/blog/2012/jan/11/defend-humanitiesgraduates.

Bertolt B. 1964. Brecht on theatre: The development of an aesthetic[M]. John Willett, Trans.. New York: Hill and Wang.

Billotte K. 2012. Conservatives killed the liberal arts[N/OL]. Salon, 2012- 09- 15[2018- 03- 25]. http://www.salon.com/ 2012/09/14/conservatives_killed_the_liberal_arts/.

Bonnell A. 2014. Humanities \& social science under attack[J/ OL]. Advocate, 21(1). http://www.nteu.org.au/article/Humanities-and-social-science-under-attack-16020.

Brindley A. 1999. The role of backyards in suburban life: A case study: Millswood, Adelaide[J]. South Australian Geographical Journal, 98: 61-77.

Campaign for Social Science. 2015. The business of people: The significance of social science over the next decade [M]. London: Sage.

Cech T R. 1999. Science at liberal arts colleges: A better edu-
cation?[J] Daedalus, 128: 195-216.

Christ C T. 2005. A world of experiences: We must prepare our students to live in a smaller world. Smith Alumnae. Spring[EB/OL]. 2015-05- 17 [2018-03-25]. http://www. smith.edu/president-christ/speeches-writings/world- experiences-spring-2005.

Christie M. 1992. Grounded and ex-centric knowledges: Exploring Aboriginal alternatives to western thinking[Z]. Paper presented at the Fifth International Conference on Thinking, Townsville, Queensland.

Coghlan C. 2014. Australia and live animal export: Wronging nonhuman animals[J]. Journal of Animal Ethics, 4(2): 4560. doi:10.5406/janimalethics.4.2.0045.

de Rosa M. 2003. Privacy in the age of terror[J]. The Washington Quarterly, 26(3): 27-41. doi:10.1162/016366003765609552.

Dean A. 2015. Japan's humanities chop sends shivers down academic spines[N/OL]. The Guardian. 2015-09-26[2018-0325]. http://www.theguardian.com/higher- education- network/2015/sep/25/japans- humanities- chop- sends- shiversdown-academic-spines.

Dear M. 1988. The postmodern challenge: Reconstructing human geography[J]. Transactions of the Institute of British Geographers, 13(3): 262-274. doi:10.2307/622990.

Deresiewicz W. 2014. Excellent sheep: The miseducation of the American elite and the way to a meaningful life[M]. New York: Free Press.

Douglas W, Neal J. 2013. Engaging the Muslim world: Public diplomacy after 9/11 in the Arab Middle East, Afghanistan, and Pakistan[M]. Boulder, CO: Rowman and Littlefield.

Else H. 2014. Students unrealistic about skill level of jobs[N/ OL]. Times Higher Education. 2014-06-26[2018-03-25]. https://www.timeshighereducation.com/news/students- unrealistic-about-skilllevel-of-jobs/2014160.article.

Faust D G. 2010. The role of the university in a changing world[Z/OL]. 2010- 06- 30[2018- 03- 25]. http://www.harvard.edu/president/speech/2010/role- university- changingworld.

Foucault M. 1988. Power/knowledge: Selected interviews and other writings, 1972-1977[M]. New York, NY: Random House.

French T, LaBerge L, Magill P. 2011. We're all marketers now [J/OL]. McKinsey Quarterly, 2011- 12- 18[2018- 03-25]. http://www.mckinsey.com/insights/marketing_sales/ were_all_marketers_now.

Fulbright J W, Tillman S P. 1989. The price of empire[M]. New York: Pantheon.

Garden R. 2015. Who speaks for whom? Health humanities and the ethics of representation[J]. Medical Humanities, 41 (2): 77-80. doi:10.1136/medhum-2014-010642. 
Gentzler J. 2003. What is a death with dignity?[J]. The Journal of Medicine and Philosophy, 28(4): 461-487. doi:10.1076/ jmep.28.4.461.15968.

Ginn F, Beisel U, Barua M. 2014. Flourishing with awkward creatures: Togetherness, vulnerability, killing[J]. Environmental Humanities, 4: 113- 123. doi:10.1215/220119193614953.

Giroux H A. 2011. Beyond the swindle of the corporate university: Higher education in the service of democracy[Z/OL]. Truthout, 2011-01-18[2018-03-25]. http://www.truth-out. $\mathrm{org} /$ opinion/item/69:beyond- the- swindle- of- the- corporateuniversity-higher-education-in-theservice-of-democracy.

Grove J. 2015. Social sciences and humanities faculties 'to close' in Japan after ministerial intervention[N/OL]. Times Higher Education, 2015-09-14[2018-03-25]. https://www. timeshighereducation.com/news/social- sciences- and- humanities-faculties-close-japan-after-ministerialintervention.

Gruen D, Kelly J, Gorecki S, et al. 2011. Wellbeing, living standards and their distribution[J/OL]. Economic Roundup, (3): 81- 98[2018- 03- 25]. http://www.treasury.gov.au/ PublicationsAndMedia/Publications/2011/Economic-

Roundup- Issue- 3/Report/Wellbeing- living- standards- andtheir-distribution.

Hall D E. 2013. The value of the liberal arts in an undergraduate education: Address to the Lehigh Tower Society[Z/ OL]. 2013-09-28[2018-03-25]. https://cas.cas2.lehigh.edu/ content/valueliberal-arts-undergraduate-education.

Hay I, Beaverstock J. 2016. Handbook on wealth and the super-rich[M]. Cheltenham, UK: Edward Elgar.

Hay I, Muller S. 2014. Questioning generosity in the golden age of philanthropy: Towards critical geographies of superphilanthropy[J]. Progress in Human Geography, 38: 635653. doi:10.1177/0309132513500893.

Head L, Muir P. 2013. Backyard[M]. Ultimo, NSW: Halstead Press.

Hohendahl P U. 2005. The future of the research university and the fate of the humanities[J]. Cultural Critique, 61(1): 1-21. doi:10.1353/cul.2005.0033.

Hopkins K. 2014. A liberal arts degree is a waste of time[N/ OL]. The Spectator, 2014-02-14[2018-03-25]. http://blogs. new.spectator.co.uk/2014/02/katie- hopkins- a- liberal- artsdegree-is-a-wasteof-time/.

Institute for the Future. 2011. Future work skills 2020[R/OL]. [2018-03-25]. http://www.iftf.org/futureworkskills/.

Lehrer J. 2011. Steve jobs: 'Technology alone is not enough' $[\mathrm{J} /$ OL]. The New Yorker, 2011-10-07[2018-03-25]. Retrieved from http://www.newyorker.com/news/news- desk/stevejobs-technology-alone-isnot-enough.

Lewin T. 2013. As interest fades in the humanities, colleges worry[N/OL]. The New York Times, 2013-10-30[2018-03-
25]. http://www.nytimes.com/2013/10/31/education/as- interest-fades-in-the-humanities-colleges-worry.html?_r=0.

Matthews D. 2013. Spinach for its own sake[Z/OL]. 2013-0425[2018- 03- 25]. https://www.insidehighered.com/news/ 2013/04/25/head-scottish-university-critiques-idea-promoting-knowledge-its-own-sake.

McLean M R. 2005. The future of food: An introduction to the ethical issues in genetically modified foods[Z/OL]. 200504[2018-03-25]. Paper presented at the conference entitled The Future of Food: Legal and Ethical Challenges held at Santa Clara University, California.

McLeod C M. 2004. Pondering nature: An ethnography of duck hunting in southern New Zealand[D/OL]. [2018-0325]. Dunedin: University of Otago, New Zealand. http:// hdl.handle.net/10523/3423.

McLoughlin K. 2014. Authoring war: The literary representation of war from the Iliad to Iraq[M]. Cambridge, England: Cambridge University Press.

Brandy Schillace. 2015. Medical humanities in conversation [Z/OL]. [2018-03-25]. http://mh.bmj.com/.

Miller J H. 2014. What ought humanists to do?[J] Daedalus, 143(1): 19-34. doi:10.1162/DAED_a_00250.

Mittelstrass J. 2015. Humanities under pressure[J]. Humanities, 4: 80-86. doi:10.3390/h4010080.

National Academies of Sciences, Engineering, and Medicine. 2015. Integration of Education in the Humanities, Science, Engineering and Medicine[R/OL]. 2015-07-27[2018-0327]. https://webcache.googleusercontent.com/search?q= cache:q0w3ewxBtQsJ:https://sites.nationalacademies.org/ PGA/bhew/humanitiesandstem/+ $\quad \& \mathrm{~cd}=1 \& \mathrm{hl}=\mathrm{en} \& \mathrm{ct}=$ clnk\&gl=au.

Newfield C. 2010. Avoiding the coming higher ed wars. American Association of University Professors[R/OL]. 2010May- June[2018- 03- 27]. https://www.aaup.org/article/ avoiding-coming-higher-ed-wars\#.WriNWYhuaUk .

Nussbaum M. 2010. Cultivating the imagination[N/OL]. The New York Times, 2010-10-17[2018-03-25]. http://www.nytimes.com/roomfordebate/2010/10/17/do- colleges- needfrenchdepartments/cultivating-the-imagination.

OECD. 2016. Future of Work in Figures[R/OL]. 2016-01-14 [2018- 03-25]. http://www.oecd.org/employment/ministerial/future-of-work-in-figures.htm.

OECD Labour and Employment Ministerial Meeting. 2016. Ministerial statement. Building more resilient and inclusive labour markets. Paris: Organisation for Economic Cooperation and Development[R/OL]. 2016-01-15[2018-0326]. http://www.oecd.org/employment/ministerial/labourministerial-statement-2016.pdf.

Owen W. 1920. Poems[M]. London: Chatto and Windus. 
PBO (Parliamentary Budget Office). 2016. Higher education loan program: Impact on the budget[R]. Canberra: Commonwealth of Australia. (Report No. 02/2016).

Phamotse M, Kissack M. 2008. The role of the humanities in the modern university: Some historical and philosophical considerations[J]. Journal of Philosophy of Education, 42 (1): 49-65. doi:10.1111/jope.2008.42.issue-1.

Preston A. 2015. The war against humanities at Britain's universities[N/OL]. The Guardian, 2015-03-29[2018-03-25]. http:/www.theguardian.com/education/2015/mar/29/waragainst-humanitiesat-britains-universities.

Romano H. 2016. A tale of two Berkeleys[N/OL]. The Daily Californian, 2016-01-13[2018-03-25]. http://www.dailycal. org/2015/10/16/a-tale-of-two-berkeleys/.

Sawa T. 2015. Humanities under attack[N/OL]. The Japan Times, 2015-08- 23[2018-03-25]. http://www.japantimes. co.jp/opinion/2015/08/23/commentary/japan- commentary/ humanitiesattack/\#.Vm4PY mqpBc.

Sayer A. 2000. Realism and social science[M]. London: Sage.

Scalia C J. 2015. Conservatives, please stop thrashing the liberal arts[N/OL]. The Wall Street Journal, 2015-03-27[201803-25]. http://www.wsj.com/articles/christopher-scalia-conservatives-pleasestop-trashing-the-liberal-arts-1427494073.

Shaw R. 2015. Opinion: BA myth-busting overdue[EB/OL]. Massey University: About. 201512-17[2018-03-25]. http:// www.massey.ac.nz/massey/about-massey/news/article.cfm? mnarticle uuid=406E0EA8-E2B1- 7AA8-C9C8-C2976D7 809D8.

Smith J K A. 2006. Who's afraid of postmodernism? Taking Derrida, Lyotard and Foucault to church[M]. Grand Rap- ids, Michigan: Baker Academic.

Soja E. 1989. Postmodern geographies: The reassertion of space in critical social theory[M]. London: Verso.

Souter D. 2010. Harvard commencement 2010[Z/OL]. 201005-27[2018- 03-25]. https://www.youtube.com/watch?v= eCxaDwOCXD8.

Starr P. 2013. Remedy and reaction: The peculiar American struggle over health care reform[M]. New Haven, CT: Yale University Press.

Terras M, Priego E, Liu A, et al. 2013. The Humanities Matter! [R/OL]. 2013-07[2018-03-26]. http://4humanities.org/wpcontent/uploads/2013/07/humanitiesmatter300.pdf.

Trimper J B, Wolpe P R, Rommelfanger K S. 2014. When "I" becomes "We": Ethical implications of emerging brain-tobrain interfacing technologies[J]. Frontiers in Neuroengineering, 7(4): 1-4. doi:10.3389/fneng.2014.00004.

UC Berkeley. 2018. Big ideas courses[Z/OL]. 2018- 03- 26 [2018-03-26]. http://bigideascourses.berkeley.edu/.

Wadhwa V, Freeman R B, Rissing B A. 2008. Education and Tech Entrepreneurship[R/OL]. 2008- 05- 01[2018- 03-25]. https://ssrn.com/abstract=1127248.

Walker P. 2015. England has highest university tuition fees in industrialised world, survey finds[N/OL]. The Guardian, 2015-11-24[2018-03-25]. http:/www.theguardian.com/education/2015/nov/24/uk-has-highest-undergraduate-tuitionfees-in-industrialised-world-survey-finds.

Wilsdon J, Walker D. Social science is vital too[N/OL]. The Guardian. 2015-12-06[2018-03-25]. http://www.theguardian.com/science/politicalscience $/ 2015 / \mathrm{feb} / 25 / \mathrm{so}-$ cialscience-is-vital-too

\title{
Defending letters:
}

\section{A pragmatic response to assaults on the humanities}

\author{
Iain HAY
}

(School of the Environment, Flinders University, Adelaide 5001, SA, Australia)

\begin{abstract}
This paper is a mainly pragmatic response to utilitarian criticisms of the humanities. It first outlines political, public and practical fronts on which the humanities are under assault, identifying critics and their conspirators. Then, as a part of its defence of the humanities it expounds some of their central strengths. These range from the philosophical to the practical: lending critical perspectives to knowledge production, enriching lives, developing skills for uncertain and increasingly connected futures, improving science and supporting institutional income and credibility. Finally, the paper suggests that humanists must not only continue valuable conceptual and empirical disciplinary work but must add the very defence of the humanities to professional activity.
\end{abstract}

Key words: arts; crisis; defending; humanities; liberal arts; utility 